\title{
El sector silvoagropecuario y su contribución al desarrollo en los principales centros nodales de la Patagonia chilena en los años 2012 y 2007
}

\author{
The agriculture and forestry sectors and their contribution to development \\ in major hubs of Chilean Patagonia in 2012 and 2007
}

Sergio Soza-Amigo ${ }^{1}$, Claudio Mancilla ${ }^{2}$, Luz María Ferrada ${ }^{3}$

\section{RESUMEN}

Utilizando tablas input-output de los años 2012 y 2007 de los principales centros nodales de la Patagonia chilena asociados a los Andes Australes (Valdivia, Osorno, Puerto Montt, Coyhaique y Punta Arenas), se analiza y contrastan los cambios, similitudes, encadenamientos e impactos que en ellas presentó el sector silvoagropecuario. Para identificar los cambios y similitudes se recurrió al índice de Le Masne (1988), para los encadenamientos la técnica de extracción hipotética presentada por Dietzenbacher y van der Linden en 1997 y, para los impactos; las modificaciones propuestas por Soza-Amigo en 2008 a los coeficientes de dispersión presentados por Rao y Harmston en 1979. La investigación responde a las siguientes preguntas: ¿existió una similitud y cambio estructural común en los centros que manifestaron una orientación hacia el sector silvoagropecuario? y, ¿los encadenamientos e impactos que emanan del sector silvoagropecuario son mayores que el promedio de las actividades? De los resultados se desprende que no hay cambios en las estructuras y que los encadenamientos e impactos del sector silvoagropecuario están por debajo del promedio del sistema económico.

Palabras clave: similitudes y cambios estructurales, vocación productiva, Patagonia.

\begin{abstract}
In this paper we analyze and compare the economic changes, similarities, linkages and impacts made by the agriculture-forestry sectors in the Chilean Patagonia's major hubs (Valdivia, Osorno, Puerto Montt, Coyhaique and Punta Arenas) between 2007 and 2012, thus using an input-output model. Changes and economic similarities were identified with the rate of Le Masne (1988), while the economic linkages were determined with the technique of Hypothetical extraction by Dietzenbacher and van der Linden (1997); similarly, the measurement of impact the economic changes was made by using the method proposed by Soza-Amigo (2008), which is a modification of the dispersion coefficients presented by Rao and Harmston (1979). The questions to answer in this research are, There was any similarity or common structural changes in the main hubs oriented to agriculture and forestry? And, Are the economic linkages and impacts from the agriculture and forestry sector higher than the average of the economic activities? According to the results, no changes were experienced by the economic structures, as the economic linkages and impacts of agriculture and forestry sectors remained under the average.
\end{abstract}

Key words: similarities and structural changes, productive vocation, Patagonia.

\section{Introducción}

Un problema que se presenta en los Centros Nodales de la Patagonia Chilena (Valdivia, Osorno, Puerto Montt, Coyhaique y Punta Arenas), es una inconsistencia entre sus "vocaciones productivas" y las "intenciones de desarrollo que se imponen". No se observa un diálogo entre las realidades productivas con las actividades que se establecen como prioritarias en las distintas estrategias de desarrollo, las que regularmente apuntan al turismo.

\footnotetext{
1 Universidad Austral de Chile, Escuela de Ingeniería Comercial, Puerto Montt, Chile.

2 Universidad de Los Lagos, Departamento de Ciencias Administrativas y Económicas, Osorno, Chile.

3 Universidad de Los Lagos, Departamento de Ciencias Administrativas y Económicas, Osorno, Chile.

* Autor por correspondencia: sergio.soza@uach.cl
} 
Lo anterior, es motivado por la presencia de atractivos naturales en las cercanías de estos centros (Santuario de la Naturaleza Carlos Andwanter (en los alrededores de Valdivia); el Parque Nacional Puyehue y el Vicente Pérez Rosales, cercanos a Osorno y Puerto Montt; la Reserva Nacional Río Simpson (Coyhaique); Torres del Paine o, Pali-Aike, en las cercanías de Punta Arenas), los que tienden a generar una suerte de "pugna en términos de planificación" con otras actividades que pudiesen aportar más dinamismo al desarrollo local.

Lo expuesto, se manifiesta en las distintas estrategias de desarrollo, donde se prioriza la actividad turística. El problema en general, es que en tales documentos no se indaga, en si aquellas propuestas son adecuadas para las estructuras económicas y la funcionalidad de cada sistema y subsistemas, o si estas son capaces de desarrollar los centros a partir de tales iniciativas.

Una contribución que ayuda a solucionar la pugna detectada es un estudio que entregue elementos que permitan identificar o validar cuál es la "vocación productiva" de cada uno de estos centros, entendiendo por ella "un conjunto de condiciones que deben articularse y estar a su vez en una sintonía natural con el fin de fomentar el desarrollo".

La importancia de detectar la "vocación productiva", se sustenta en que presenta un conjunto de información que ayuda a entender cómo las distintas actividades se ven involucradas (SozaAmigo, 2016a). La información obtenida contribuiría a la elaboración de políticas en la medida que es detectada con técnicas que; identifican sus impactos, cuantifican su importancia, velocidad y alcance con que transmiten sus repercusiones al sistema productivo.

Otro aspecto que potencia el desarrollo de un centro, es la sintonía entre su estructura y un entramado mayor como el de la economía regional o nacional a la que pertenece. Esto último, dadas las repercusiones que puedan tener las posibles políticas de desarrollo que se apliquen, puesto que, en general, ellas tienen un alcance regional o nacional y en pocas ocasiones están focalizadas.

La literatura plantea que las actividades que promueven el desarrollo y que impactan en el mismo serían pocas. Si existe una estructura jerarquizada y articulada, se favorece la trasmisión de los distintos efectos que de ellas emanan. Las posibles diferencias o repercusiones serían atribuibles al rol que en ellas juegan las actividades que utilizan altos y medianos niveles de tecnología (AlatristeContreras, (2015); Duman y Ertar, (2015); Revilla et al., (2015); Alatriste-Contreras y Fagiolo, (2014); De Miguel et al., (2014); Luo, (2013a y 2013b); Lopes et al., (2012); García et al., (2008); Hidalgo et al., (2007); Aroche-Reyes, (2006); Hewings et al., (1988); y Hewings, (1984)).

La información que identifica la "vocación productiva" de un centro nodal, se puede resumir en cuatro aspectos: 1) una estructura productiva que promueva el desarrollo en forma expansiva y rápida; 2) una estructura laboral coincidente con el entramo productivo, en especial, en economías que se basan en commodities con altos excedentes brutos de explotación, en comparación a los pocos puestos de trabajos que en ellas se generan o los salarios que se cancelan; o bien, estructuras laborales y productivas disonantes pero que promuevan el desarrollo y no sean concentradoras de actividad; 3) una conmutación neta que tienda a cero, como proxy del equilibrio del mercado laboral $\mathrm{y}$; 4) una estructura en línea con el desarrollo económico de la región.

Solucionar lo planteado ayudará a focalizar políticas de organización espacial que apunten a mejorar la gestión de los territorios. De esta forma, se apoyaría la gestión para una futura implementación de políticas regionales, que sean acorde a lo que se manifiesta en cada zona e integradas a una visión de desarrollo mayor (regional o nacional).

En la Figura 1 se representan los PIB per cápita de cada centro perteneciente a la Patagonia chilena. Sus incrementos ayudan a visualizar la senda y dinámica que presenta dicha variable. También se observa como han ido todos a excepción del PIB de Punta Arenas en aumento. La razón de tal disminución se debería a la escasez de gas natural en la zona, insumo para otras industrias.

Desde una perspectiva sectorial, la Figura 2 representa la distribución de las principales participaciones por ramas de actividad en la zona. El sector silvoagropecuario (compuesto por las actividades de productos agrícolas, frutas, ganado, productos pecuarios y carnes, productos silvícolas, madera y papel) ha sido importante en la Patagonia en especial si se contrasta con las actividades propias del turismo (comercio, restaurantes y hoteles y, transporte y comunicaciones; parte baja y central del gráfico, respectivamente). 


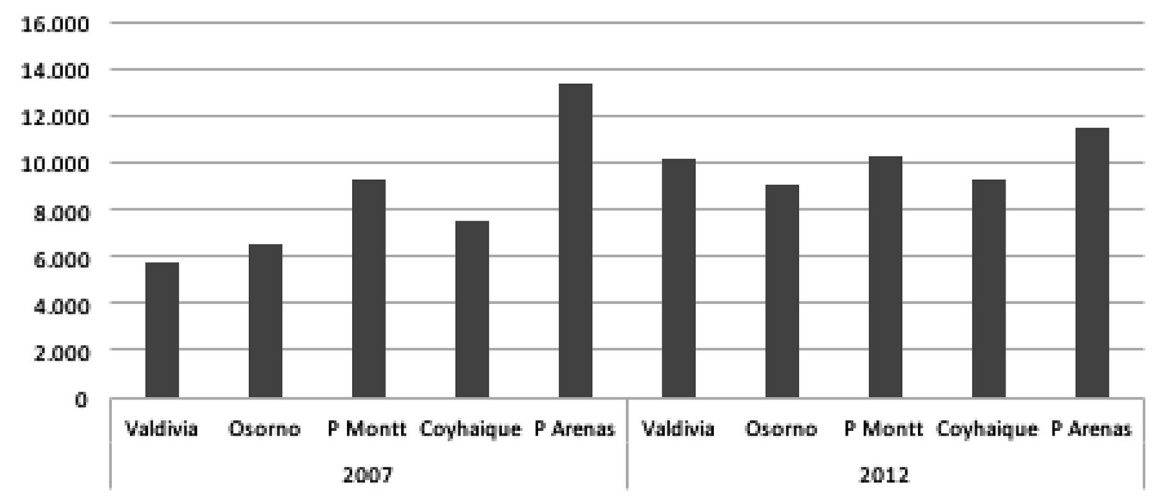

Figura 1. PIB per cápita en dólares de cada año (Fuente: Propia).

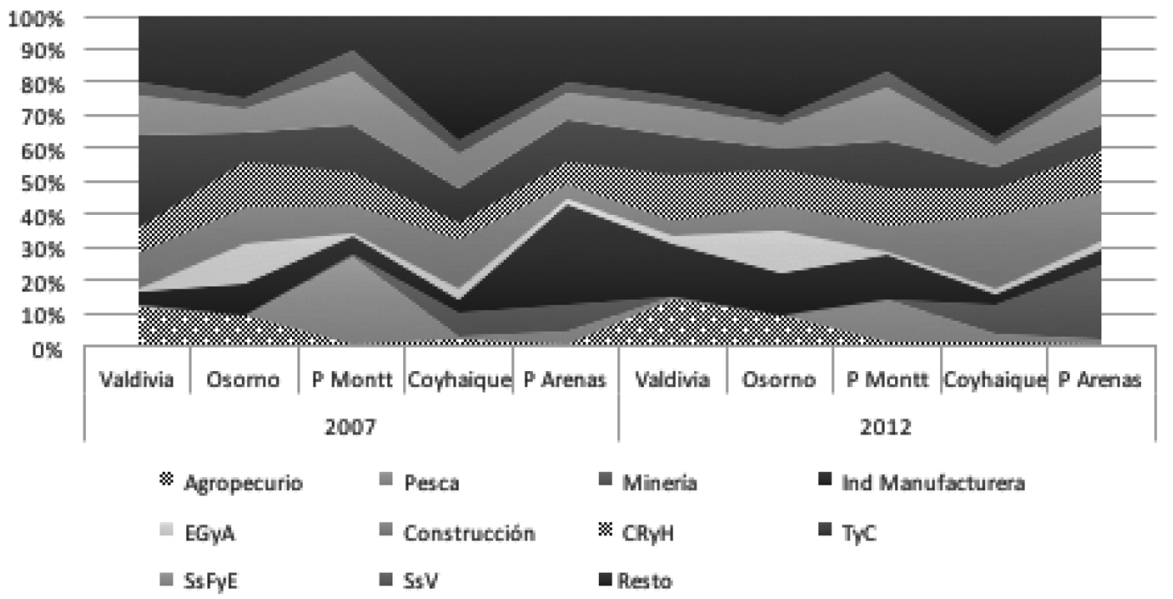

Figura 2. Participación de las actividades en el PIB de las regiones de la Patagonia. Donde: EGyA (Electricidad, Gas y Agua); CRyH (Comercio, Restaurantes y Hoteles); TyC (Transporte y Comunicaciones) y, SsFyE (Servicios Financieros y Empresariales). (Fuente: Propia).

El sector silvoagropecuario, desde el año 2007 al 2012, muestra una importante participación, en la parte norte de la Patagonia. También se observa que las actividades asociadas al turismo son importantes, en Puerto Montt, Coyhaique y Punta Arenas. Sin embargo, de acuerdo con información recogida de distintas cuentas satélites e incorporadas a matrices input-output, sumado a estudios del mercado laboral y, basados en la creación de emprendimientos, este sería bajo en la zona patagónica (Soza-Amigo, (2016a y 2016b); Zuñiga et al., (2015); y, Soza-Amigo y Maripani, (2011)).

En resumen, se presenta un escenario en donde se visualizan dos actividades que dadas las condiciones y particularidades de la zona podrían contribuir al desarrollo; entonces, la pregunta a resolver es: ¿cuál impacta y ha impactado más en su desarrollo?

Con el fin de identificar la "vocación productiva" de cada centro en la Patagonia chilena, en este trabajo, aplicando el índice de similitud estructural de Le Masne (1988), se analizaron y contrastaron los cambios y semejanzas que estos presentaron y, el de estos con la economía chilena utilizada como patrón de referencia; adicionalmente, se revisa cómo influyó en términos de relaciones (encadenamientos) e impacto (alcance) el sector silvoagropecuario y el turismo en la zona.

Los principales hallazgos permiten concluir que entre los centros existe una alta heterogeneidad, que son muy dispares respecto a la evolución de la economía chilena y, que el sector silvoagropecuario ha ido perdiendo importancia en la zona norte. 


\section{Metodología}

Utilizando las matrices input-output de Valvidia, Osorno, Puerto Montt, Coyhaique y Punta Arenas, de los años 2007 y 2012 (matrices domésticas obtenidas con la técnica RAS y, corregidas mediante coeficientes de localización) y, la de los años 2008 y 2012 para Chile (Banco Central de Chile), el trabajo que se desarrolló se dividió en dos etapas. Primero, se detectó la similitud estructural a partir del índice de Le Masne (1988) desde dos perspectivas, una referida a las estructuras y funciones de producción entre los centros nodales y; una segunda, donde nos preguntamos cómo ha sido el cambio del centro respecto al cambio que experimentó Chile.

Identificando los encadenamientos hacia atrás (enfoque de demanda; BL) y adelante (enfoque de oferta; FL), se respondió a cuáles son las actividades "motoras" del dinamismo. La técnica utilizada fue la de extracción hipotética desarrollada por Dietzenbacher y van der Linden en 1997 (ver en Anexo). La idea que existe tras tal propuesta es preguntarse: ¿qué ocurre si cierta actividad se elimina del sistema económico?

Luego, siguiendo la propuesta denominada de Rasmussen-Hirschman se jerarquizan las actividades. Una rama será "clave", si tanto su BL como FL están por sobre la media; "impulsora", si solo lo está su BL; "base", si es su FL el que está por sobre el promedio e, "independiente o isla", si sus BL y FL están bajo este último.

Para identificar la propagación de los efectos se hace uso de los coeficientes de dispersión propuestos por Rao y Harmston en 1979 pero modificados posteriormente por Soza-Amigo en 2008. Interesa indagar si los requerimientos y oferta que hace una actividad están bajo o sobre el promedio. Si una actividad que aumenta su demanda final, presenta un índice de dispersión $\left(v_{. j}\right)$ bajo, indicará que tiene un efecto amplio en términos de dispersión. Si presenta un $v_{i}$ bajo, implicará que la actividad depende en forma homogénea del sistema de industrias.

Luego de relativizar los impactos, se clasificaron en; "de alto impacto", "de demanda", "de oferta" y "bajo impacto", de acuerdo con la dispersión que ellos manifestaron.

Con el fin de realizar una comparación en conjunto entre los resultados de las similitudes obtenidas con el índice de Le Masne y la que se recoge mediante los índices de dispersión ponderados, se utilizó la técnica de clúster jerárquico. Para la obtención se definen los distintos impactos como variables y los centros como casos, esto es, se conglomera por variables. Definidos estos, se tipificó la información, obteniendo así rangos comparables.

Dado que el análisis de clúster se realizó con la técnica de Ward, como primer paso para cotejar resultados se utiliza una "matriz de distancias", a menor distancia existirá mayor similitud, la medida utilizada es la Euclídea al cuadrado $\left[d(i ; j)^{2}=\Sigma_{k}\left(x_{i k}-x_{j k}\right)^{2}\right]$. Obtenidos estas, se agrupan los centros, donde además se ha incluido a la economía chilena, para usarla como referencia de los distintos impactos e importancia de los mismos.

\section{Resultados}

En relación a las similitudes estructurales se destaca lo siguiente:

1. En el caso de Chile se observó una alta similitud entre los años 2012 y 2008. En 4 años la economía cambió un escaso $6,49 \%$, lo que acusa un estancamiento en términos globales de las funciones de producción.

2. Para el año 2007, las estructuras de los distintos centros respecto a Chile presentaron una similitud promedio próxima al 78,00\%. Con unos máximos y mínimos de 77,87 (Chile-08 con Osorno-07) y 73,03\% (Chile-08 con Puerto Montt-07), respectivamente. En general, los centros se parecen más entre sí que, respecto a la estructura global que presentó Chile para esa fecha.

3. En 2012, las similitudes de los centros respecto a Chile, en general, fue la misma y osciló entre $78,95 \%$ y $72,53 \%$, siendo el promedio de $75,50 \%$, en tal sentido se aprecia que los centros respecto al año base, se han ido retrasando en términos de competitividad.

4. Valdivia, fue el centro que presentó más similitud con Chile, tanto para los años 2007-08 y 2012, logrando una semejanza del 73,72 y 78,95\%, respectivamente.

5. En general, los centros respecto al patrón de referencia presentaron estructuras y dinámicas distintas; sin embargo, ello no implica que no estén cambiando.

6. Las variaciones en las estructuras de los centros según magnitud fueron; Punta Arenas (27,96\%), 
Valdivia (22,58\%), Puerto Montt (19,60\%), Osorno $(18,49 \%$ ) y Coyhaique con un $10,61 \%$.

7. Valdivia, Osorno y Puerto Montt son similares, situación parecida se daría para Coyhaique y Punta Arenas, en conclusión, la Patagonia chilena presentó dos grupos estructurales.

Al comparar las estructuras de los centros del año 2007 con la de Chile del año 2012, se obtiene a modo de proxy las diferencias que se requerían realizar en las funciones de producción de los centros desde el año 2007 al 2012 con el fin de acercarlas a las que logró el patrón de referencia en el año 2012. Una mayor similitud se asoció a menores cambios requeridos en el centro nodal. En esta lógica, solo Valdivia requería implementar menores cambios en el sector primario, $11,47 \%$ en concreto en la actividad productos silvícolas, luego Osorno con $13,18 \%$ en ganados, productos pecuarios y carne. La industria manufacturera, en general, requería de mayores cambios, el centro que menos modificaciones debía realizar era Coyhaique en lo referido a industria metálica. Con respecto a los servicios, se requieren menores cambios.

Asumiendo que Chile es eficiente, se revisó las similitudes que mostraban los centros en el año 2012 respecto a este. Las mayores similitudes para el sector primario se dan en Valdivia y Osorno. En el sector manufacturero no se observaron semejanzas importantes, por el contrario, los menores valores son precisamente en este ítem. El sector servicios, es el que presenta más cantidad de sectores similares y, serían coincidentes para todos los centros, la razón se debería a que no existen procesos productivos dependientes de tecnología avanzada, observándose la mayor similitud en el sector educación (los 5 centros mostraron una similitud sobre 90\%). Solo Valdivia presentó un segundo sector con similitud superior al $90 \%$ (Electricidad; 90,55\%).

Para identificar las actividades más dinámicas, se recurre a la evolución que presentó cada centro entre los años 2012 y 2007 (Cuadro 1 del anexo). Una menor semejanza se interpreta como un mayor cambio hacia la búsqueda de un mejor proceso productivo. Las actividades que más cambiaron resultaron ser; en el sector primario, la minería tanto en Valdivia, Osorno como Puerto Montt, en Coyhaique el sector pesquero y en Punta Arenas el sector frutas. Para el sector industrial se observó más coincidencias, siendo el único centro que cambió en lo referido a la industria no metálica, Punta Arenas. El resto presentó cambios en la industria metálica. Finalmente, en el sector servicios, todos coincidieron en el sector electricidad, la excepción fue Coyhaique que cambió menos en servicios comerciales.

Si bien existió concordancia en los sectores que más se modificaron, las magnitudes de los cambios fueron disímiles, por ejemplo, en el sector industrial metálico, el centro que más cambió fue Valdivia $(48,92 \%)$ y el que menos se modificó fue Coyhaique (21,85\%), estos valores, dan luz acerca de las diferencias productivas que existían en el año 2012, por un lado, en la propia dinámica y, por otro, asumiendo que inicialmente tenían las mismas funciones de producción en sus mejoras.

En relación al sector silvoagropecuario y turismo, se observa que:

1. En la zona la primera actividad es muy dinámica respecto a lo que ocurre en promedio en Chile. Mientras en este último se mantiene con variaciones cercanas al 5\%, en los centros son más acentuados. El principal cambio se observó en Punta Arenas para el sector frutas (48,59\%) y el menor en Coyhaique para los productos agrícolas (10\% aproximadamente).

2. En comparación a las actividades del turismo, algunos centros cambian más en el sector silvoagropecuario que en el primero (Puerto Montt, Coyhaique y Punta Arenas), otros lo harían más en turismo que en el sector silvoagropecuario (Valdivia y Osorno).

3. El patrón de comparación presentó un cambio en el sector turismo cercano al $6 \%$, indicando que la parte norte de la Patagonia está evolucionando y/o potenciándose hacia este. La parte sur-austral cambió hacia ambos sectores (turismo y sector silvoagropecuario), pero principalmente hacia el sector silvoagropecuario. En términos de modificaciones, los cambios observados en la Patagonia, manifiestan una evolución hacia el sector turismo.

Revisados los encadenamientos, llamó la atención que el sector silvoagropecuario fuese en su mayoría independiente, tanto en la Patagonia como en Chile. Solo se detectó una excepción y fue en el año 2012 en Puerto Montt. En promedio las actividades del sector silvoagropecuario no son relevantes en términos de la demanda u oferta 
de insumos o productos, desde y hacia el resto del sistema económico. Si hipotéticamente tales actividades y productos, así como relaciones que forman son eliminadas del sistema, las distintas economías no se verían sustancialmente afectadas. La excepción sería el caso de Puerto Montt, donde se generaría un daño, por medio del sector frutas (s-02) dada la importancia que el mismo presentó en el entramado económico del año 2012.

Basados en variadas cuentas satélites o informes anuales para el turismo (ver INE, 2013) o Sernatur (serie 2003 a 2006), donde se señala que el turista; en general, consume en los sectores: comercio, hoteles y restaurantes, transporte terrestre, marítimo y aéreos, servicios y comunicaciones, entre otros, planteamos el supuesto que la totalidad del gasto en todos los sectores señalados, corresponderá a turismo. Tal supuesto obedece a que los cambios en los flujos de consumo son motivados por turistas, por tanto, asumir aquello sirve como proxy entre lo que consume el mercado doméstico y los turistas. Lo anterior, se utiliza como forma alternativa y aproximada para determinar el impacto del sector turismo en términos de las relaciones interindustriales que se forman en estos centros nodales.

En el caso de las actividades vinculadas al turismo, estas mostraron una importancia variada. En conjunto son relativamente importantes, en especial en la parte norte de la Patagonia. El turismo afectó más y en forma similar a Valdivia y Osorno en los años 2012 como 2007. En el caso de Puerto Montt y Coyhaique, estas actividades eran más importantes en el año 2007, por desgracia para el año 2012, se observó un retroceso de ellas, a tal punto, que las actividades quedan desconectadas del desarrollo de tales centros. Para Punta Arenas, se observó un avance desde el año 2007 al 2012; sin embargo, la actividad característica del sector (hoteles y restaurantes), era apenas base en 2012, no mostrando una conexión superior al promedio con el resto del sistema económico por medio de la compra de insumos. Finalmente, en el caso de Chile, no se observó un cambio en la relevancia de dichas actividades, siendo base el comercio y clave restaurantes y hoteles.

La parte norte de la Patagonia se vería más afectada en su desarrollo por el turismo que por las actividades ligadas a la actividad silvoagropecuaria. En general, se observó que, tanto en Chile como en la Patagonia, el sector silvoagropecuario no fue gravitante en los años 2007 y 2012.

$\mathrm{Si}$ los resultados de los encadenamientos se complementan con sus respectivos coeficientes de dispersión, se observa que el sector silvoagropecuario en Valdivia, Osorno y Puerto Montt, presentó para ambos años los mayores impactos e incluso si estos se comparan con los de Chile.

En el caso de Valdivia, se hace evidente desde el punto de vista de la propagación de los impactos la importancia que tiene el sector silvoagropecuario. Por otra parte, en Osorno, si bien el sector agrícola (s-01) en el año 2007 era uno que solo impactaba en un sentido de demanda, en el 2012 lo era en ambos, por su parte, en el de frutas (s-02), ocurrió lo contrario.

En Puerto Montt, los impactos son altos; dos casos interesantes fueron la agricultura y frutas; para el primero en 2007 presentaba bajo impacto y en 2012 pasó a tener uno alto y, en el caso de frutas, estas perdieron relevancia pasando de ser de alto impacto a tener solo un impacto de oferta. Para Coyhaique, el sector silvoagropecuario, presenta una propagación solo en un sentido de demanda. En Punta Arenas, el sector en general es de bajo impacto, situación similar ocurriría en Chile, con la excepción del sector agrícola (s-01) y productos silvícolas, maderas y papel (s-04), que del año 2007 al 2012 pasaron de tener bajo impacto a uno que afecta en un sentido de oferta.

En el caso de las actividades del turismo los impactos son más homogéneos y, en general, son importantes en la Patagonia y en Chile. En el caso de Valdivia, tanto en 2007 como en 2012, todas las actividades que forman parte de este fueron de alto impacto. En Osorno, los sectores de hotelería y restaurantes (s-14) y servicios conexos al transporte (s-17), presentaron impactos de demanda solamente en ambos años. Para Puerto Montt, los impactos son algo más variados pero bajos, así los sectores hotelería y restaurantes (s-14) y servicios conexos al transporte (s-17) terminaron siendo de bajo a nulo impacto, situación similar se vio en Coyhaique con la salvedad que estos sectores manifestaron un impacto del tipo de oferta. Finalmente, tanto en Punta Arenas como en Chile, el turismo es de alto impacto, en un sentido de propagación de sus relaciones.

Se puede establecer que en términos de impacto y de contraste entre el sector silvoagropecuario y turismo, que la vocación productiva de la Patagonia, 
está dividida. Los impactos para el primero son distintos en la parte norte y sur, en concordancia con las características de la zona (norte; sector silvoagrepecuario y sur, sector ganadero). En el caso del turismo, afectaría en forma homogénea y alta a estos centros.

En resumen, para la parte norte, solo Valdivia manifiesta altos impactos en ambos sectores. Para Osorno y Puerto Montt, si bien estos son altos en general, los vinculados al turismo no son tan influyentes como en Valdivia. En Coyhaique, el sector silvoagropecuario, es menos gravitante que el turismo, situación similar se observa para Punta Arenas y Chile.

Realizado lo anterior, se procedió a encontrar las similitudes en términos de impacto por medio de un clúster jerárquico, en Anexo el Cuadro 2 hace referencia a un diagrama de témpanos vertical y, finalmente y también an Anexo el Cuadro 3, a un dendrograma entregrupos.

Los resultados señalan una coincidencia en las similitudes de las estructuras y estas, a su vez, con la zona geográfica donde los centros están insertos. Las comunas de Valdivia y Osorno son similares en términos de estructuras y propagación de sus impactos. Situación similar se observó entre Puerto Montt y Coyhaique, con la salvedad que sus similitudes estructurales son menores que las que manifiestan Valdivia y Osorno. Para el año 2012 estas últimas se parecían en 91,52\% mientras que Puerto Montt y Coyhaique en 83,98\%. El caso de Punta Arenas es diferente, en términos estructurales no presenta altas similitudes con los centros ni con Chile, su mayor valor lo logra con Coyhaique $(86,58 \%)$, pero en términos de impacto su similitud la logra con Chile, lo que respondería a las actividades ausentes en los otros centros.

Algunas pautas que nos arrojan los resultados que permitirían responder a qué centro se desarrolla más y en cierta forma conviene imitar son;

1. La estructura del patrón cambió $6,49 \%$, el centro que más cambió fue Punta Arenas $(27,96 \%)$.

2. La actividad que más varió en Chile fue gas y agua (s-11) y, en Valdivia, Osorno y Puerto Montt la minería (s-07), en Coyhaique los pescados y productos del mar industrializados (s-05) y, en Punta Arenas la industria manufacturera no metálica (s-08).
3. En el caso del sector silvoagropecuario, las mayores similitudes con respecto a Chile en el año 2012, se dieron en Valdivia y Osorno, siendo los sectores frutas (s-02); ganado; productos pecuarios y carne (s-03) y pescados y productos del mar (s-05), los que más coincidieron.

4. Para el turismo, se observó que el mayor número de actividades semejantes y las mayores similitudes entre actividades se dieron en Valdivia, Osorno y Puerto Montt.

5. El centro que presentó la mayor similitud con Chile en el año 2012 resultó ser Valdivia y Osorno con unas semejanzas de 78,95 y 78,73\%, respectivamente. Las actividades que más se parecieron con el patrón fueron electricidad (s-10; 90,55\%) y educación (s-23; 94,03\%) para Valdivia y para Osorno, educación (s-23; 89,99\%).

6. Desde la perspectiva de los encadenamientos, Chile presentó seis actividades clave en ambos años, Valdivia se mantuvo en 7; Osorno de 5 pasó a 6; Puerto Montt de 7 retrocedió a 2, en Coyhaique se retrocedió de 6 a 4 y en Punta Arenas se incrementó de 1 a 7 .

7. En Chile solo existió una actividad clave en el sector primario en 2012 (minería; s-07), en la que coincidieron Coyhaique (año 2007) y Punta Arenas (año 2012).

8. La industria manufacturera no es gravitante en Chile; sin embargo, lo es en la Patagonia.

9. Los sectores construcción (s-12); hotelería y restaurantes (s-14); administración pública (s-22); educación (s-23) y salud (s-24) son clave en Chile, en los centros en general existe coincidencia en estos sectores, la excepción sería el sector hotelería y restaurantes (s-14).

10. En términos de actividades de alto impacto, en Chile se incrementaron de 14 a 15 , los centros las disminuyeron, Valdivia (de 21 a 17), Osorno (de 15 a 14), Puerto Montt (de 17 a 16), Coyhaique (de 14 a 12) y Punta Arenas de 17 a 16.

11. En términos de similitud con los impactos se forman tres grupos Chile con Punta Arenas, Puerto Montt con Coyhaique y Valdivia con Osorno.

En general existe una coincidencia con los resultados referidos al índice de Le Masne y los encadenamientos, en especial con el grupo formado por Osorno y Valdivia. 


\section{Conclusiones}

Asumiendo un escenario que presenta cambios en el ámbito de la tecnología e innovación, los mismos se debieran reflejar en los procesos productivos y en su estructura global. En tal lógica, el índice de Le Masne mostró que la economía chilena -la que se toma como referencia- presentó un cambio del 6,5\% desde el año 2008 al 2012. Los centros manifestaron cambios por casi el doble y en algunos casos hasta más de cuatro veces, ello es indicativo de la importancia del cambio tecnológico que se experimenta en la Patagonia.

En general las estructuras de los centros se asemejan a la estructura de Chile, tanto en el año 2012 como en 2008 con un valor próximo al $75 \%$. Esto es, las estructuras de los centros tienen baja semejanza con el patrón de referencia; sin embargo, mostraron cambios importantes en sus funciones de producción, lo que se entiende las acerca más a niveles competitivos.

Revisados los encadenamientos y la dispersión de los efectos, se observó que en general, hay coincidencia con las similitudes detectadas con el índice de Le Masne. Por otra parte, las actividades que resultaron ser clave e impulsoras, no presentaron en los centros importantes efectos de dispersión hacia el resto del sistema, tanto en un sentido de demanda como de oferta. Es decir, pueden presentar encadenamientos tanto en un sentido de demanda como oferta e incluso en un sentido más clásico solo de demanda, pero la propagación de efectos es baja, por tanto, manifiestan que sus repercusiones son más bien acotadas.

Utilizada la técnica de clúster jerárquico, se detectó que las estructuras de la parte norte de la Patagonia (Valdivia y Osorno) resultaron ser los más semejantes en términos productivos y de impactos. La segunda similitud, se encontró para los centros de la parte central (Puerto Montt y Coyhaique). La particularidad es que aun cuando presentaron una similitud estructural, ella es menor que la de los centros de la parte norte. Solo quedo marginado de estas similitudes Punta Arenas, que presentó semejanza con la distribución de impactos de Chile.

En general, las actividades propias del sector silvoagropecuario, son más características en la parte norte, y que estas son más semejantes respecto a Chile en el año 2012, esto es, sus estructuras son semejantes entre sí y, estas simultáneamente con la de Chile y, adicionalmente, presentan impactos en este sector similares.

En el caso del turismo, en términos de difusión de sus efectos al resto del sistema tanto en términos de demanda $\left(\mathrm{v}_{\mathrm{j} . \mathrm{j}}\right)$ como de oferta $\left(\mathrm{v}_{\mathrm{i}}\right)$, son importantes y sus efectos son similares en los centros y en Chile.

Finalmente, responder a qué centro se desarrolla o qué actividades interactúan más con el resto del sistema económico se hace complejo, ya que en ninguno se presentó concordancia en los diferentes aspectos evaluados. En tal lógica, se esperaría que los centros de la parte norte de la Patagonia incrementaran más su desarrollo dadas las similitudes que mostraron con el patrón de referencia en especial motivados por los sectores silvoagropecuario y turismo; sin embargo, el resto de las actividades en tales centros no presentan un entramado común con el país.

Lo último, se hace más evidente al comparar las distintas demandas y ofertas de insumos o productos y, evidentemente en las relaciones que emanan de ellas. En tal sentido, el turismo que se desarrolla en la parte norte de la Patagonia, pareciera ser que contribuye más al conjunto de la economía como un todo, y no solo al desarrollo de los centros como tal.

Desde el punto de vista de los impactos, si bien, hay centros que bajaron sus actividades más articuladoras, en general se mantienen.

En resumen, existen una serie de factores a considerar. En este trabajo, se apuesta por lo logrado en la parte norte de la Patagonia, donde existe una fuerza común entre los encadenamientos y los impactos de las relaciones que afectan más a los distintos centros.

\section{Agradecimientos}

Se agradece a Fondecyt por los financiamientos entregados por medio de la investigación: "La Vocación Productiva, el Nivel Educativo y la Funcionalidad del Centro Emisor y Receptor como Factores Explicativos de la Conmutación Inter e Intrarregional en la Región de los Andes Australes de la Patagonia Chilena", Proyecto No 1160196. Además, informamos que versiones preliminares de este trabajo se presentaron en la XVIII Reunión de Economía Mundial, Universidad de Alcalá de Henares, España, 2016 y, en la XLII Reunión de Estudios Regionales, Universidad de Santiago de Compostela, España, 2016. 


\section{Literatura Citada}

Alatriste-Contreras, $\mathrm{M}$.

2015. The Relationship Between the Key Sectors in the European Union Economy and the Intra-European Union Trade. Journal of Economic Structures, 4 (14): 1-24.

Alatriste-Contreras, M. and G. Fagiolo

2014. Propagation of Economic Shocks in Input-Output Networks: A cross-country analysis. LEM: LEM Working Papers Series, April, 2014/09.

Aroche-Reyes, F.

2006. Trees of the Essential Economic Structures: A qualitative input-output method. Journal of Regional Science, 46 (2): 333-353.

De Miguel, J.; M. Llop and A. Manresa

2014. Sectoral Productivity Gains in Two Regional Economies: Key sectors from a supply-side perspective. The Annals of Regional Science, 53 (3): 731-744.

Dietzenbacher, E. \& J. van der Linden

1997. Sectoral and Spatial Linkages in the EC Production Structure. Journal of Regional Science, 37 (2): 235-257.

Duman, A. and G. Ertan

2015. Input-Output Networks and Growth Performances Across Countries. Working Papers in Economics, november, $15 / 04$.

García, A., A. Morillas and C. Ramos

2008. Key Sectors a New Proposal from the Network Theory. Regional Studies, 42 (7): 1013-1130.

Hewings, G., R.C. Jensen and G. R. West 1988. Fields of Influence of Technological Change in InputOutput Models. Papers of Regional Science Association, 64: 25-36.

Hewings, G.

1984. The Role of Prior Information in Updating Regional InputOutput Models. Socio-Economic Planning, 18 (5): 319-336.

Hidalgo, C., B. Klinger, A.-L. Barabási and R. Hausmann 2007. The Product Space Conditions the Development of Nations. Science, 317: 482-487.

Hirschman, A.

1958. The Strategy of Economic Development. New Haven, Connecticut, USA, Yale University Press.

Instituto Nacional de Estadísticas, (INE), Chile

(2013). Informe Anual del Turismo 2013. Disponible en Internet: http://www.ine.cl.

Le Masne, $\mathrm{P}$.

1988. Le système productif français face a ses voisins européens, Troisième Colloque de Comptabilité Nationale, Paris, 12-14 décembre.

Lopes, J., J. Dias and J. Amaral.

2012. Assessing Economic Complexity as Interindustry Connectedness in Nine OECD Countries. International Review of Applied Economics, 26 (6): 811-827.
Luo, J.

2013a. The Power-of-Pull of Economic Sectors: Acomplex networks analysis, Complexity, 18 (5): 37-47.

Luo, J.

2013b. Which Industries to Bail Out First in Economic Ression? Ranking US Industrial sectors by the Power-ofPull. Economic Systems Research, 25 (2), 157-169.

Miller, R. and P. Blair

2009. Non Survey And Partial- Survey Methods: Fundamentals. In: Input-Output: Analysis Foundations and Extensions. Cambridge University Press, pp. 303-346, pp. 750.

Rasmussen, P.

1956. Studies in Inter-Sectoral Relations. Amsterdam, North- Holland P. C.

Rao, V. and F. Harmston

1979. Identification of Key Sectors in a Region of a Developed Economy. Annals of Regional Science, 13 (3): 78-90.

Revilla, D., A. García-Ándres and I. Sánchez-Juárez.

2015. Identification of Key Productive Sectors in the Mexican Economy. Expert Journal of Economics, 3 (1): 22-39.

\section{SERNATUR}

2008. Cuentas Satélite de Turismo Serie 2003 a 2006. Santiago: Servicio Nacional del Turismo.

Soza-Amigo, S.

2016a. Conmutación Centrípeta-Centrífuga: ¿es la vocación productiva una motivación para conmutar? Idesia, 34 (1): 55-67.

Soza-Amigo, S.

2016b. Impactos del Turismo y de la Conmutación CentrípetaCentrífuga en el Sur de Chile: Enfoques Clásicos y Nuevos para su Interpretación. Revista Métodos Cuantitativos para la Economía y Empresa, 21: 58-76.

Soza-Amigo, S.

2008. Relaciones Intersectoriales en Magallanes: En busca de su estructura económica. Magallania, 36 (1): 79-102.

Soza-Amigo, S. y L. Correa

Regiones Extremas Chilenas y su Invisibilidad Económica. Si Somos Americanos. Revista de Estudios Transfronterizos, XIV (2): 187-216.

Soza-Amigo, S. y J. Maripani

2011. The Input-Output Matrix of the Punta Arenas City: Description, analysis and simulation. Magallania, 39 (1): 103-118.

Zuñiga-Jara, S., S. Soza-Amigo y K. Soria-Barreto

2015. Dinámica del Emprendimiento y el Desempleo en la Patagonia Chilena. Magallania, 43 (1): 103-115. 


\section{ANEXOS}

Índice de Le Masne (1988):

$S^{a-b}=100\left[1-\frac{1}{2} \sum_{i=1}^{n+1}\left|a_{i j}^{a}-a_{i j}^{b}\right|\right] \rho_{d} ; \operatorname{con} \rho_{d}=\frac{\left(x_{i j}^{n}+x_{i j}^{(n-1)}\right)}{\left(\sum_{i} x_{i j}^{n}+\sum_{i} x_{i j}^{(n-1)}\right)}$

Donde:

$a_{i j}=$ Coeficiente técnico que se define como: $x_{i j} / X_{j}$, con $a_{i j} \in A$ (matriz de coeficientes técnicos).

$n=$ Corresponde al número de sectores o regiones.

$X=$ Representa a la producción total.

$x_{i j}=$ Corresponde a sus inputs.

Extracción hipotética presentada por Dietzenbacher y van der Linden (1997):

$B L_{j}^{D-v d L}=\left[\frac{i^{t}\left(x-\bar{x}_{j}(k)\right)}{x_{j}}\right] 100 \quad F L_{i}^{D-v d L}=\left[\frac{x^{t}\left(x-\bar{x}_{i}^{t}(k)\right) i}{x_{i}}\right] 100$

Donde:

$k$ : Hace alusión a la actividad que ha sido extraída.

$\mathrm{i}^{\mathrm{t}}$ : Vector unitario y " $\mathrm{t}$ " que se trata de una transposición.

Soza-Amigo (2008; basado en Rao y Harmston (1979)):

$v_{j}^{w}=\frac{\sqrt{\frac{1}{n-1} \sum_{i=1}^{n}\left(z_{i j}-w z_{. j}\right)^{2}}}{w z_{. j}} ; \operatorname{con} j=1,2, \ldots, n \quad v_{i}^{w}=\frac{\sqrt{\frac{1}{n-1} \sum_{j=1}^{n}\left(g_{i j}-w g_{. i}\right)^{2}}}{w g_{i .}} ; \operatorname{con} i=1,2, \ldots, n$

Donde:

$v j^{w}:$ C. D. Ponderado; con $j=1,2, \ldots, n \mathrm{y}, z_{i j} \in \mathrm{Z}=(\mathrm{I}-\mathrm{A})^{-1}$.

$v_{i}{ }^{w}$ : C. D. Ponderado; con $i=1,2, \ldots, n$, y $g_{i j} \in \mathrm{G}=(\mathrm{I}-\mathrm{B})^{-1}$.

$b_{i j}$ : Elemento de la matriz de distribución; $b_{i j}=x_{i j} / X_{i}$, con $b_{i j} \in \mathrm{B}$ (matriz de distribución).

$x$ : Output (producción total).

$w$ : Ponderador; $Y i / y$ (economías pequeñas) y $\mathrm{Xi} / \mathrm{x}$ (economías desarrolladas). 
Cuadro 1. Cambios en las funciones de producción para cada Centro y Chile.

\begin{tabular}{|c|c|c|c|c|c|c|c|c|}
\hline & Sector & & $\begin{array}{l}\text { Valdivia- } \\
0712\end{array}$ & $\begin{array}{c}\text { Osorno- } \\
0712\end{array}$ & $\begin{array}{l}\text { P. Montt- } \\
0712\end{array}$ & $\begin{array}{l}\text { Coyhaique- } \\
0712\end{array}$ & $\begin{array}{c}\text { Punta Arenas- } \\
0712\end{array}$ & $\begin{array}{l}\text { Chile- } \\
0812\end{array}$ \\
\hline \multirow{7}{*}{$\begin{array}{l}\text { Sector } \\
\text { primario }\end{array}$} & s-01 & Productos agrícolas & 78,67 & 84,76 & 58,18 & 90,24 & 73,90 & 93,38 \\
\hline & s-02 & Frutas & 84,94 & 84,25 & 82,97 & 83,61 & 51,41 & 96,79 \\
\hline & s-03 & Ganado; productos pecuarios y carne & 78,92 & 79,60 & 78,48 & 85,10 & 69,32 & 92,51 \\
\hline & s-04 & Productos silvícolas; maderas y papel & 78,33 & 79,47 & 70,51 & 82,37 & 74,01 & 95,63 \\
\hline & s-05 & Pescados y productos del mar & 77,57 & 80,41 & 83,31 & 80,12 & 59,91 & 90,45 \\
\hline & s-06 & Petróleo & - & - & - & - & 70,85 & 90,37 \\
\hline & s-07 & Minería & 50,04 & 45,56 & 46,51 & 87,44 & 67,94 & 95,20 \\
\hline \multirow{2}{*}{$\begin{array}{l}\text { Sector } \\
\text { industrial }\end{array}$} & s-08 & Industria manufacturera no metálica & 70,86 & 73,36 & 83,50 & 83,82 & 41,77 & 95,12 \\
\hline & s-09 & Industria manufacturera metálica & 51,08 & 63,28 & 73,15 & 78,15 & 53,83 & 86,38 \\
\hline \multirow{19}{*}{$\begin{array}{l}\text { Sector } \\
\text { servicios }\end{array}$} & s-10 & Electricidad & 51,19 & 67,67 & 63,83 & 89,45 & 46,87 & 87,92 \\
\hline & s-11 & Gas y agua & 74,35 & 78,61 & 79,15 & 94,19 & 76,57 & 78,15 \\
\hline & s-12 & Productos de la construcción & 68,57 & 73,68 & 68,70 & 89,08 & 63,31 & 93,50 \\
\hline & s-13 & Servicios comerciales & 70,38 & 78,79 & 81,59 & 84,12 & 73,44 & 96,34 \\
\hline & s-14 & S. de hotelería y de restaurantes & 69,07 & 74,72 & 68,88 & 90,30 & 67,27 & 93,95 \\
\hline & s-15 & Transporte ferroviario y caminero & 70,59 & 78,01 & 77,89 & 87,44 & 64,31 & 95,50 \\
\hline & s-16 & Transporte marítimo y aéreo & 68,14 & 71,87 & 72,67 & 89,38 & 62,03 & 93,19 \\
\hline & s-17 & Servicios conexos de transporte & 82,73 & 83,37 & 86,41 & 92,45 & 78,05 & 93,83 \\
\hline & s-18 & Servicios de comunicaciones & 87,02 & 91,94 & 94,86 & 91,14 & 91,49 & 89,77 \\
\hline & s-19 & Servicios financieros y de seguros & 88,33 & 93,37 & 90,75 & 92,75 & 93,59 & 97,62 \\
\hline & $\mathrm{s}-20$ & Servicios empresariales & 86,86 & 91,45 & 90,00 & 92,17 & 85,37 & 95,93 \\
\hline & s-21 & Servicios inmobiliarios y de vivienda & 96,99 & 98,82 & 97,09 & 98,46 & 91,28 & 97,49 \\
\hline & s-22 & Servicios de administración pública & 85,38 & 89,24 & 89,72 & 93,92 & 83,52 & 97,01 \\
\hline & s-23 & Educación pública y privada & 92,15 & 94,64 & 92,89 & 95,46 & 92,86 & 97,91 \\
\hline & s-24 & Servicio de salud pública y privada & 92,47 & 91,85 & 91,78 & 92,57 & 85,16 & 97,09 \\
\hline & s-25 & Esparcimiento y otros servicios & 85,21 & 89,05 & 87,30 & 93,58 & 82,84 & 96,67 \\
\hline & & Máximo & 96,99 & 98,82 & 97,09 & 98,46 & 93,59 & $\mathbf{9 7 , 9 1}$ \\
\hline & & Promedio & 77,42 & 81,51 & 80,40 & 89,39 & 72,04 & $\mathbf{9 3 , 5 1}$ \\
\hline & & Mínimo & 50,04 & 45,56 & 46,51 & 78,15 & 41,77 & 78,15 \\
\hline
\end{tabular}

Fuente: Propia. 
Cuadro 2. Diagrama de témpanos vertical

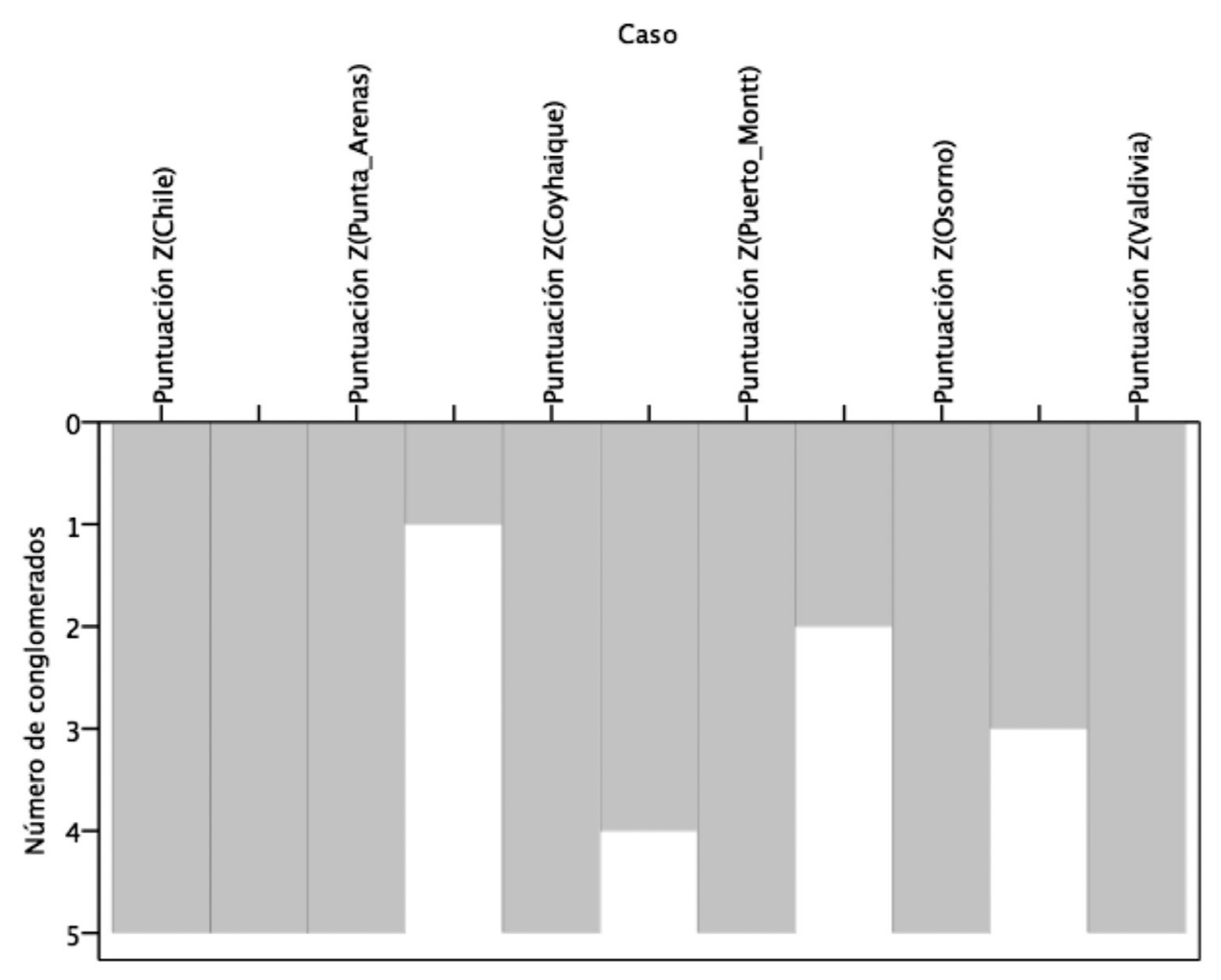

Fuente: Propia.

Cuadro 3. Dendrograma con información tipificada.

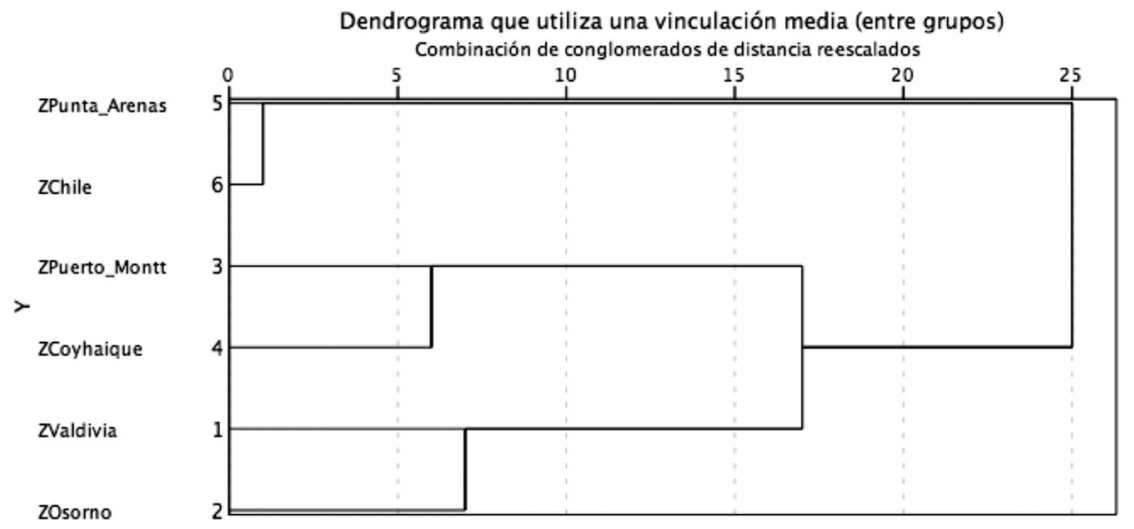

Fuente: Propia. 